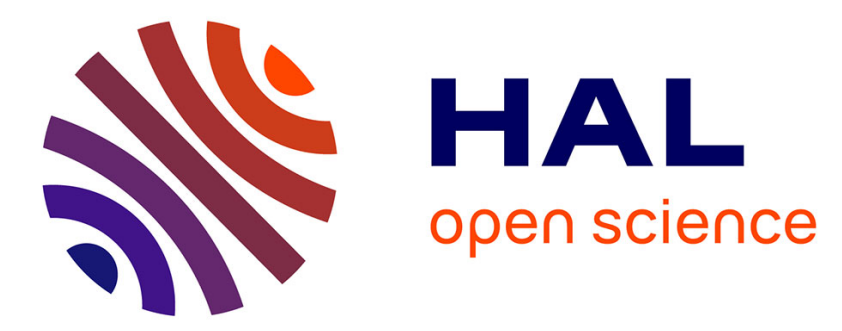

\title{
Crossover to strange metal phase: quantum criticality in one unit cell $\mathrm{Bi} 2 \mathrm{Sr} 2 \mathrm{CaCu} 2 \mathrm{O} 8+\mathrm{x}$
}

Edoardo Sterpetti, Johan Biscaras, Andreas Erb, Abhay Shukla

\section{To cite this version:}

Edoardo Sterpetti, Johan Biscaras, Andreas Erb, Abhay Shukla. Crossover to strange metal phase: quantum criticality in one unit cell $\mathrm{Bi} 2 \mathrm{Sr} 2 \mathrm{CaCu} 2 \mathrm{O} 8+\mathrm{x}$. Journal of Physics: Condensed Matter, 2020, 32 (4), pp.045601. 10.1088/1361-648X/ab4b21 . hal-02385283

\section{HAL Id: hal-02385283 \\ https://hal.sorbonne-universite.fr/hal-02385283}

Submitted on 28 Nov 2019

HAL is a multi-disciplinary open access archive for the deposit and dissemination of scientific research documents, whether they are published or not. The documents may come from teaching and research institutions in France or abroad, or from public or private research centers.
L'archive ouverte pluridisciplinaire HAL, est destinée au dépôt et à la diffusion de documents scientifiques de niveau recherche, publiés ou non, émanant des établissements d'enseignement et de recherche français ou étrangers, des laboratoires publics ou privés. 


\title{
Crossover to strange metal phase: quantum criticality in one unit cell $\mathrm{Bi}_{2} \mathrm{Sr}_{2} \mathrm{CaCu}_{2} \mathrm{O}_{8+\mathrm{x}}$
}

\author{
Edoardo Sterpetti, ${ }^{1}$ Johan Biscaras, ${ }^{1}$ Andreas Erb,${ }^{2}$ and Abhay Shukla ${ }^{1, *}$ \\ ${ }^{1}$ Institut de Minéralogie, de Physique des Matériaux et de Cosmochimie, \\ Sorbonne Université, UMR CNRS 7590, MNHN, \\ IRD UMR 206, \& Place Jussieu, F-75005 Paris, France \\ ${ }^{2}$ Walther Meissner Institut fur Tieftemperaturforschung, \\ Bayerische Akademie der Wissenschaften, \\ Walther-Meissnerstr. 8, D-85748 Garching Germany
}

(Dated: September 27, 2019)

\begin{abstract}
Transport measurements can be used to determine the phase diagram of high temperature superconductors by detecting variations in temperature dependence of resistance in different regions of the phase diagram. While for bulk measurements several samples with varying chemical doping are used, we continuously vary carrier density in our ultra-thin two-dimensional $\mathrm{Bi}_{2} \mathrm{Sr}_{2} \mathrm{CaCu}_{2} \mathrm{O}_{8+\mathrm{x}}$ device by electrostatic means and the space charge doping method. Here we concentrate on a low-disorder, high quality single unit cell thick sample. We establish the crossover to strange metal from the pseudogap and Fermi liquid phases in the normal state, close to the superconducting dome. By extrapolation we demarcate a critical doping region which is thought to correspond to a quantum phase transition at very low temperature.
\end{abstract}




\section{INTRODUCTION}

The determination of the phase diagram of $\mathrm{Bi}_{2} \mathrm{Sr}_{2} \mathrm{CaCu}_{2} \mathrm{O}_{8+\mathrm{x}}(\mathrm{BSCCO}-2212)$ and in particular the crossover lines between different regions in the vicinity of the superconducting dome is important because of the possible existence of a Quantum Critical Point (QCP) [1-3]. This QCP is thought to demarcate a quantum phase transition occurring at $0 \mathrm{~K}$ upon variation of a tuning parameter [4-9]. The tuning parameter here is the charge carrier density. In BSCCO-2212 the pseudogap to strange metal phase boundary and the strange metal to Fermi liquid crossover, common to the phase diagram of all high temperature superconductors, are not well-established. In the pseudogap phase at low doping, an energy range close to the Fermi level exhibits a paucity of available states and is effectively gapped at low temperatures. The strange metal phase at higher doping shows metallic decrease of resistance with temperature in the normal state but with a linear dependence, even at low temperatures where in conventional metals Fermi-liquid theory predicts a $T^{2}$ dependence due to the predominance of electron-electron scattering. The strange metal to Fermi liquid crossover has been rarely studied [10-13] probably due to the difficulty in fabricating sufficiently overdoped samples and adapted methods. Several methods, spectroscopic or otherwise, have been used to investigate and detect the opening of the pseudogap (occurring at temperature $T^{*}$ ) as a function of doping. Among these we can cite Angle Resolved Photo-Emission Spectroscopy (ARPES) [14], Raman spectroscopy [15], tunneling spectroscopy [16-18], c-axis resistivity [19] and Inelastic Magnetic Neutron Scattering (MINS) [20].

For a direct identification of the critical regime [8] one needs to measure the crossovers down to very low temperatures approaching $0 \mathrm{~K}$ as a function of doping and precisely determine the corresponding sheet carrier density at each doping value. Transport data cannot directly access an eventual QCP masked by the superconducting state unless superconductivity is completely suppressed by a very high magnetic field [21, 22]. Indirectly however, the crossover lines found above the superconducting dome can indicate the doping range where criticality could occur. This requires multiple and precise temperature dependent transport measurements around the optimal doping value and as close as possible to the superconducting region as well as an interpretation of the transport data in terms of variations in 
temperature dependence in different regions of the phase diagram. The position of the QCP is an important point of discussion for theories and experiments seeking to explain this phase diagram and the physics of high temperature superconductors and some even postulate the existence of two such points. Many works $[2,8,9]$ argue that this point is characteristic of the normal state strange metal phase and as such should be found at the extrapolation of the boundaries delimiting this phase in the normal state. Carrier density determination in these compounds is generally based on an empirical and commonly used relation [23] which relates the critical temperature $T_{\mathrm{c}}$ to doped holes $(p)$ per $\mathrm{Cu}$ atom (holes/ $\mathrm{Cu}$ ). Additionally one can also measure the Hall coefficient $R_{\mathrm{H}}$ and determine a Hall carrier density $n_{\mathrm{H}}=$ $1 / q R_{\mathrm{H}}$. In strongly correlated electronic systems such as cuprates the Hall estimation does not directly give the value of the carrier density as in conventional metals, but a quantity related to it. It is also temperature dependent confirming that the complex underlying electronic structure cannot be interpreted as a single parabolic band. Nevertheless as we showed earlier [24], 1/qR $R_{\mathrm{H}}$ at fixed low temperature is a useful relative indicator of carrier density modulation [21, 25-27]. In what follows the carrier density is that given by the empirical formula unless specified otherwise.

In a previous work [24] we established a comprehensive phase diagram of two-dimensional (2D) BSCCO-2212 as a function of doping and of disorder by achieving a large variation in doping on each side of the superconducting dome by means of our original space charge doping method $[28,29]$. In this work we focus on the underlying physics of the intrinsic phase diagram and concentrate on the positioning of the extrapolated quantum critical point near the top of the superconducting dome in the critical region of the phase diagram using a low disorder, 1 unit cell (u.c.) thick sample. It should be noted that other methods have been used to dope surfaces of superconductors by in situ depostion of atoms [30], ionic liquids [31] or ozone annealing [32]. Any electrostatic doping technique is efficient over a thin 2D layer determined by the screening length. In BSCCO-2212, at a thickness of 2.5 u.c. [24], discrepancies can be seen due to decreasing efficiency of the technique over the whole sample thickness, which are avoided in 1 u.c. samples. Transport measurements are affected by disorder which changes both the absolute value of the measured sheet resistance and its dependence on temperature [24]. In general several different samples are used for phase diagram measurements when doping is changed chemically. This involves stoichiometric changes which by definition introduce defects. Space charge doping, as opposed to chemical 
doping, does not affect the crystal at the atomic level and does not introduce disorder. The defect level in our single sample stays constant over all measurements since no change of stoichiometry is involved. This is an obvious advantage over a changing defect level which can induce changes in scattering rates and mechanisms over and above a change in the physical phase of the sample.

\section{SAMPLE FABRICATION}

The superconducting 2D BSCCO-2212 single crystal device is sample E with $T_{\mathrm{c}}\left(p_{\text {opt }}\right)=$ $80 \mathrm{~K}$. Some measurements from sample D of ref.[24] (maximum critical temperature $\left.T_{\mathrm{c}}\left(p_{\text {opt }}\right)=81 \mathrm{~K}\right)$ are also included to guarantee consistency. Both devices are $3 \mathrm{~nm}(1$ u.c.) thick and were fabricated on $0.5 \mathrm{~mm}$ thick soda-lime glass substrates by the anodic bonding technique [33, 34]. This technique, which uses the electrostatic attraction of a

surface space charge induced in the glass substrate for the adherence of flakes of layered materials placed on the substrate, allows us to obtain high quality samples with lateral dimensions of about $100 \mu \mathrm{m}$. Sample thickness is verified by atomic force microscopy and samples are shaped mechanically with a micromanipulator tip to isolate the area of the uniform required thickness. The large lateral size is essential because we use shadow stencil masks aligned on the sample to thermally evaporate gold contacts in a van der Pauw configuration. We thus avoid chemical degradation and surface contamination from standard electron beam lithography processes, which in our experience degrade few-unit-cell thick BSCCO-2212 crystals, eventually making them insulating [24]. Transport and Hall measurements were performed in a high-vacuum cryostat coupled to an external electromagnet. This set-up allows us to vary the temperature in the 3-400 $\mathrm{K}$ range as well as to apply a magnetic field of up to $\pm 2 \mathrm{~T}$. 


\section{RESULTS}

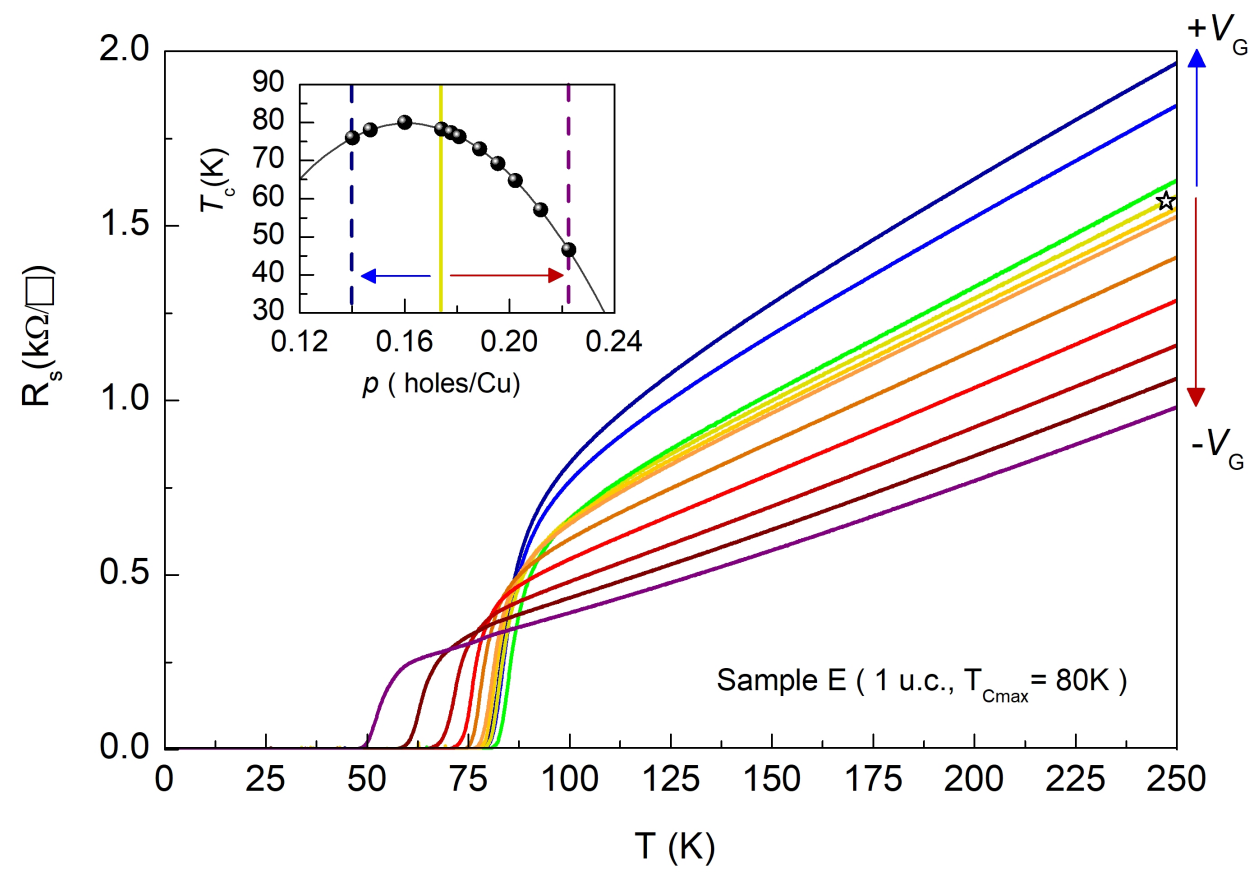

Figure 1: Space charge doping of sample E. $R_{\mathrm{S}}(T)$ curves of sample E at varying doping levels controlled by the gate voltage $V_{\mathrm{G}}$. The curve corresponding to the initial doping is indicated with a star. The $R_{\mathrm{S}}$ curve corresponding to the optimal doping level is green. The device has been doped such that holes are reversibly added and removed. The inset shows the corresponding critical temperatures shown as a function of the doping level as determined using the empirical method. The vertical lines indicate the initial doping value and the extremes attained through space charge doping while the arrows indicate the hole and electron doping ranges achieved.

Doping is tuned in the device by means of our original space charge doping method $[24,28,29]$ by monitoring the sheet resistance $R_{\mathrm{S}}$ at the doping temperature $T_{\mathrm{d}}=350 \mathrm{~K}$ while applying a gate voltage $V_{\mathrm{G}}$ of the order of $100-280 \mathrm{~V}$ between the device and a metallic electrode on the opposite face of the glass substrate. The temperature activates slight ionic mobility of sodium ions inherent to soda-lime glas and the electric field engenders their drift. This creates an ionic space charge at the glass-device interface, consisting of an excess or of a depletion layer of sodium ions. The space charge in turn induces a corresponding mirror charge in the device which can reach very high values, in the region of $10^{15} \mathrm{~cm}^{-2}$ 
because of the intense associated electric field. Lowering the temperature below $T_{\mathrm{d}}$ freezes the accumulated space charge at the interface by annulling ionic mobility in the glass substrate, thus fixing the carrier density in the device. The change in carrier density is due to the space charge for the conditions given above and not due to ion intercalation from the glass substrate or eventually oxygen diffusion in the sample[24, 28, 29]. For a given carrier density the temperature dependence of $R_{\mathrm{S}}$ is measured from $330 \mathrm{~K}$ down to well below $T_{\mathrm{c}}$.

Fig. 1 shows the sheet resistance of sample $\mathrm{E}$ as a function of temperature for varying charge carrier density. The curve corresponding to the initial doping level is marked by the star. Negative and positive gate voltages $V_{\mathrm{G}}$ were applied to increase and decrease the charge density and therefore the critical temperature $T_{\mathrm{c}}$, allowing us to reversibly [24] sweep between the overdoped regime and the underdoped regime as illustrated by the inset of Fig. 1. For these measurements the doping regime is tuned around the optimally doped region where the $T$-linear behavior of $R_{\mathrm{S}}(T)$ extends to the lowest temperatures, narrowly separating the pseudogap phase and the Fermi-liquid phase [2, 3, 35, 36].

The strange metal phase is characterized by a linear dependence of $R_{\mathrm{S}}(T)$ [35]. This linear dependence changes in other parts of the phase diagram because of different scattering regimes depending on the relative importance of inelastic and elastic scattering [7]. In BSCCO for example there is a downward deviation in the pseudogap phase from the linear dependance of the strange metal phase. To determine the phase boundaries we proceed as shown in Fig. 2 by detecting the temperature corresponding to deviation from linear dependence of $R_{\mathrm{S}}(T)$ [24]. We use a linear fit for the strange metal region with $R_{\mathrm{S}}(T)=$ $R_{0}+A T$, where $R_{0}$ is the residual sheet resistance and $A$ is the slope [2]. We define $T^{*}$ and $T_{\mathrm{m}}$ as the temperatures below which the resistance shows a downward (for $T^{*}$ ) and upward (for $T_{\mathrm{m}}$ ) deviation from the high temperature $T$-linear behavior. More precisely, $T_{\mathrm{m}}$ in the overdoped region for each doping level was extracted as the temperature above which the $R_{\mathrm{s}}(T)$ measurement could be fitted with a linear component and below which it was fitted with the power law $R_{s}(T)=R_{0}+B T^{m}$. Though a $T^{2}$ behaviour typical of the Fermi liquid is expected in the highly overdoped region, earlier experiments in cuprates have established that in the moderately overdoped phase the upward deviation of $R_{\mathrm{s}}(T)$ below $T_{\mathrm{m}}$ also follows such a power law and that the exponent $m$ varies between 1 and 2 depending on the material and the doping level [36-38]. In our overdoped devices we observed this $m(p)$ dependence 


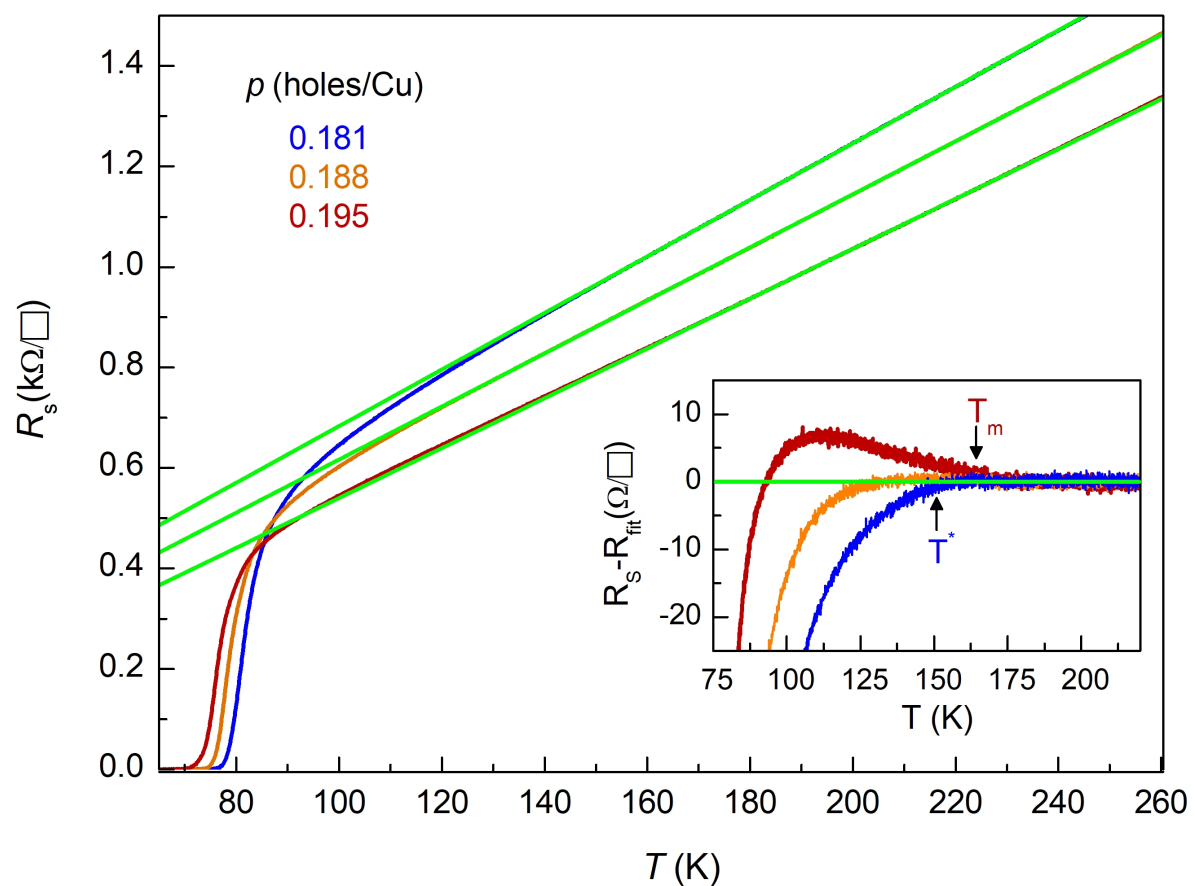

Figure 2: Linear fit of the high temperature part of the sheet resistance $R_{\mathrm{S}}(T)$ for sample E corresponding to the three doping levels indicated at the top left. The green lines are the linear fit. The inset shows the difference between $R_{\mathrm{S}}(T)$ and the linear fit for each curve from which $T^{*}$ and $T_{m}$ can be deduced as the temperatures at which deviation from linearity occurs.

[38], with for example $m=1.35$ at the doping level of $1.6 \times 10^{15} \mathrm{~cm}^{-2}(\sim 0.21$ holes $/ \mathrm{Cu})$.

$R_{\mathrm{S}}(T)$ curves at three specific doping values are shown in Fig. 2. These correspond to the lowest $T^{*}$ and $T_{\mathrm{m}}$ temperatures that we could measure on the crossover boundaries as well as one doping value in between where the $R_{\mathrm{S}}(T)$ dependence remains strictly linear till near the superconducting transition. The green lines are the linear fits to each curve and the inset shows the difference between $R_{\mathrm{S}}(T)$ and the linear fit. The blue curve corresponds to the highest doping level ( $p=0.181$ holes $/ \mathrm{Cu}$ ) where a downward deviation from linearity distinct from superconducting fluctuations is observed. The red curve corresponds to the lowest doping level ( $p=0.195$ holes $/ \mathrm{Cu}$ ) where an upward deviation from linearity is measured. The orange curve $(p=0.181$ holes $/ \mathrm{Cu})$ remains linear until the superconducting transition as the downturn of $R_{\mathrm{S}}(T)$ observable at around $110 \mathrm{~K}$ can be attributed to the superconducting fluctuation regime.

The resulting phase diagram is shown in Figure 3. The pseudogap to strange metal 


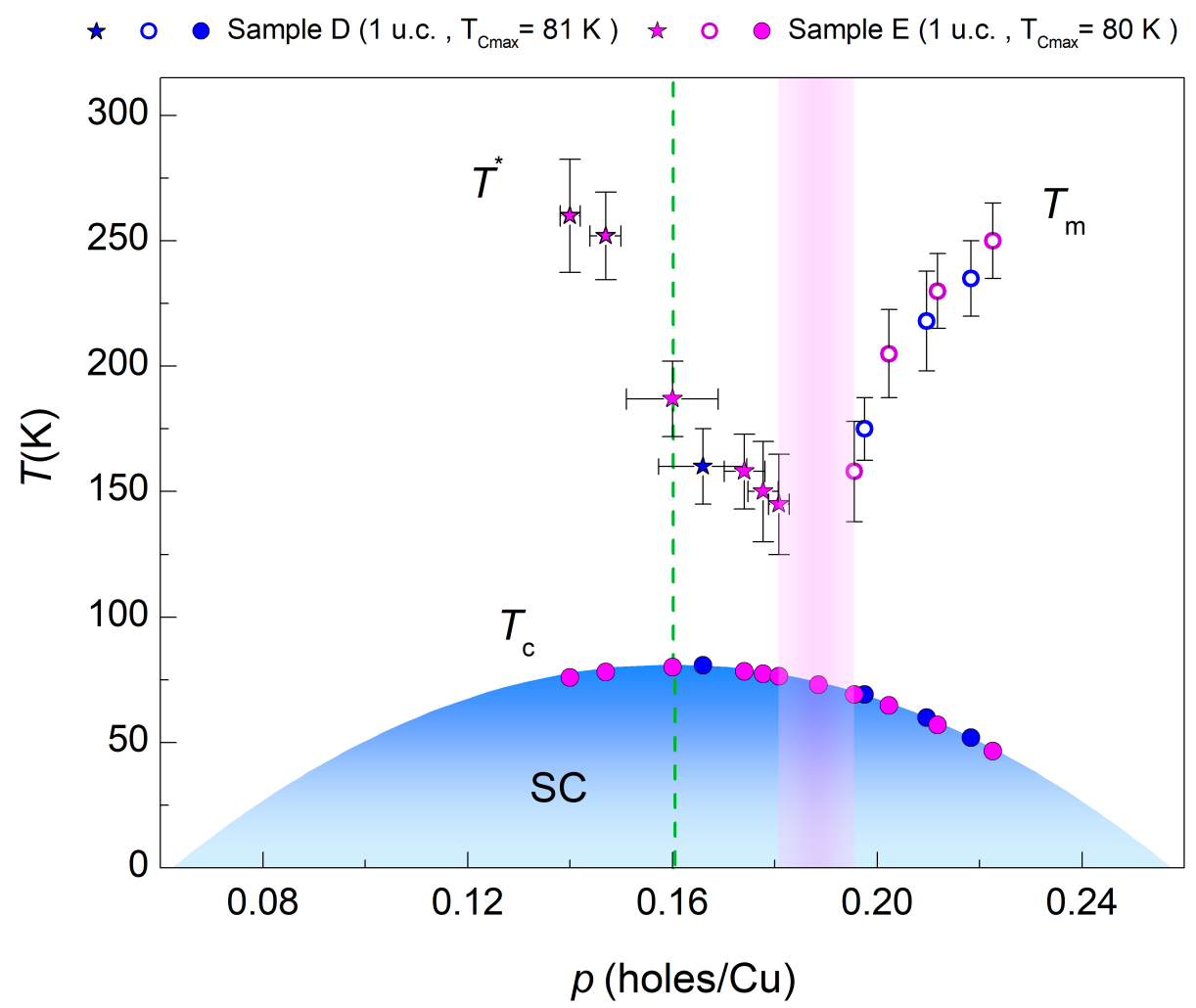

Figure 3: Phase diagram of 1 u.c.-thick low disorder BSCCO-2212. Doping dependence of the characteristic temperatures $T^{*}$ (filled symbols), $T_{\mathrm{m}}$ (empty symbols) and $T_{\mathrm{c}}$ (filled symbols on the superconducting dome) of samples D and E. The dashed vertical line indicates the optimal doping level and the violet band indicates the critical region corresponding to the low temperature strange metal phase.

crossover $T^{*}(p)$ at low temperature roughly decreases linearly as doping increases with the lowest measured temperature point clearly on the overdoped side of the phase diagram at about 0.181 holes/Cu. This recalls results found on bulk BSCCO-2212 in earlier works [1420]. The strange metal to Fermi liquid crossover $T_{m}(p)$ increases as doping increases with the lowest measured temperature point on this boundary being at a doping of 0.195 holes $/ \mathrm{Cu}$. Between these two doping values $R_{\mathrm{S}}(T)$ dependence is linear and corresponds to the strange metal phase. 


\section{DISCUSSION}

The QCP identified with a quantum phase transition is often situated at the extrapolated junction in the superconducting dome and at zero temperature, of the two phase boundaries delimiting the strange metal region $[8,9]$. In other words if these phase boundaries can be measured to the lowest possible temperatures close to the superconducting dome, the narrow strange metal region between them will provide an indication of the doping range in which the QCP could exist. By this criterion and following our measurements we identify this critical region to be in the range 0.181-0.195 holes/ $\mathrm{Cu}$, inside the dome corresponding to the superconducting region. We can thus conclude that this normal state strange metal region at low temperatures extrapolates to what could be a quantum critical point inside the superconducting dome, in agreement with recent findings [39, 40]. We note that this identification has been possible both by the use of high quality low-disorder samples and by accurately measuring the temperature dependence of sheet resistance at a stabilized low rate of $0.3-0.5 \mathrm{~K} / \mathrm{min}$. These precautions reduce extrinsic influence which could alter the temperature dependence of $R_{\mathrm{S}}(T)$ and influence the determination of these phase boundaries. Thus we have been able to distinguish the pseudogap and Fermi liquid related deviations of $R_{\mathrm{S}}(T)$ from linear behavior to below $150 \mathrm{~K}$.

Several spectroscopic measurements [14-18, 20] have been carried out on bulk samples with charge carrier density varied by controlling oxygen doping. They have mostly identified the critical region by investigating the underdoped part of the phase diagram. They seem to agree on a linear decrease of $T^{*}$ with doping from the underdoped side to the optimal central part of the dome $(p=0.16$ holes $/ \mathrm{Cu})$, within the substantial latitude permitted by large error bars. Some of them revealed a subsequent flattening on the slightly overdoped side, followed by an abrupt collapse at larger doping [20] with a QCP estimated at $p^{*}=0.19$ holes $/ \mathrm{Cu}$.

As discussed above, $n_{\mathrm{H}}=1 / q R_{\mathrm{H}}$ as measured from the Hall effect cannot be directly related to the carrier density in these materials but is a useful relative indicator of carrier density modulation. In contrast with the situation in simple metals $R_{\mathrm{H}}$ in this case is temperature dependent. Hall measurements at a few temperatures and doping levels in one of our BSCCO-2212 samples are shown in Fig. 4. The $R_{\mathrm{H}}$ vs $T$ curves corresponding to different doping levels in Fig. 4a confirm that the maximum of $R_{\mathrm{H}}(\mathrm{T})$ is found at $T=120 \mathrm{~K}$ and 

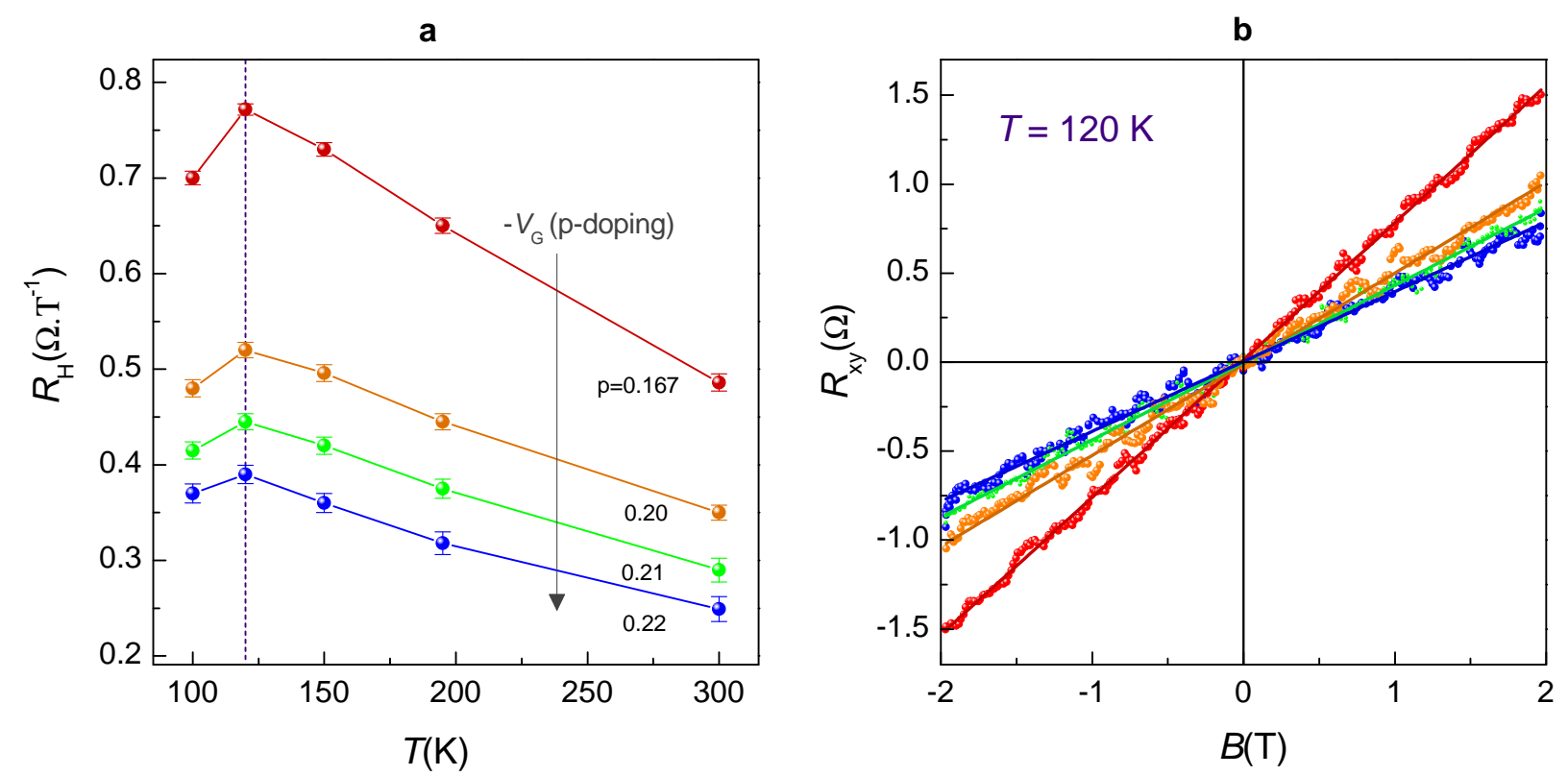

Figure 4: Hall measurements. a, Temperature dependence of the Hall coefficient $R_{\mathrm{H}}$ for different doping levels tuned by the external gate voltage $V_{\mathrm{G}}$. $\mathbf{b}$, Transverse anti-symmetrized resistance $R_{\mathrm{xy}}$ as a function of magnetic field at $T=120 \mathrm{~K}$. Colors correspond to the same doping level in the two panels.

that the variation of $R_{\mathrm{H}}$ with temperature decreases as doping is increased from optimal to the overdoped[26], approaching the Fermi liquid part of the phase diagram. We use $R_{\mathrm{H}}$ at $120 \mathrm{~K}$ to calculate the Hall carrier density $n_{\mathrm{H}}$. Fig. $4 \mathrm{~b}$ shows the anti-symmetrized transverse resistance $R_{\mathrm{xy}}$ as a function of applied magnetic field $B$ at $T=120 \mathrm{~K}$.

The phase diagram of Fig. 3 for samples $\mathrm{D}$ and $\mathrm{E}$ with carrier density inferred by $R_{\mathrm{H}}$ is shown in Fig. 5. The range of critical doping as estimated through $1 / q R_{\mathrm{H}}$ is $[0.88$ $1.1] \times 10^{15} \mathrm{~cm}^{-2}$. Despite the difficulty in relating $1 / q R_{\mathrm{H}}$ directly to the doping level, an advantage of this approach is that the error in the determination of $1 / q R_{\mathrm{H}}$ is relatively constant $\left( \pm 2-4 \times 10^{13} \mathrm{~cm}^{-2}\right)$ over the whole doping range, being related only to the determination of the slope of the Hall voltage. In contrast the error in the doping value determined by the empirical formula is maximum in the optimal doping region of the superconducting dome. This is because the dome is flat in this region, so that relatively large variations of the charge carrier concentration produce small variations of critical temperature. This is seen in the size of the horizontal error bars in Fig. 3 and in Fig. 5. Specifically, an error of $\pm 1 \mathrm{~K}$ in the determination of $T_{\mathrm{c}}$ results in an error on $p= \pm 0.01$ holes $/ \mathrm{Cu}$ around the 


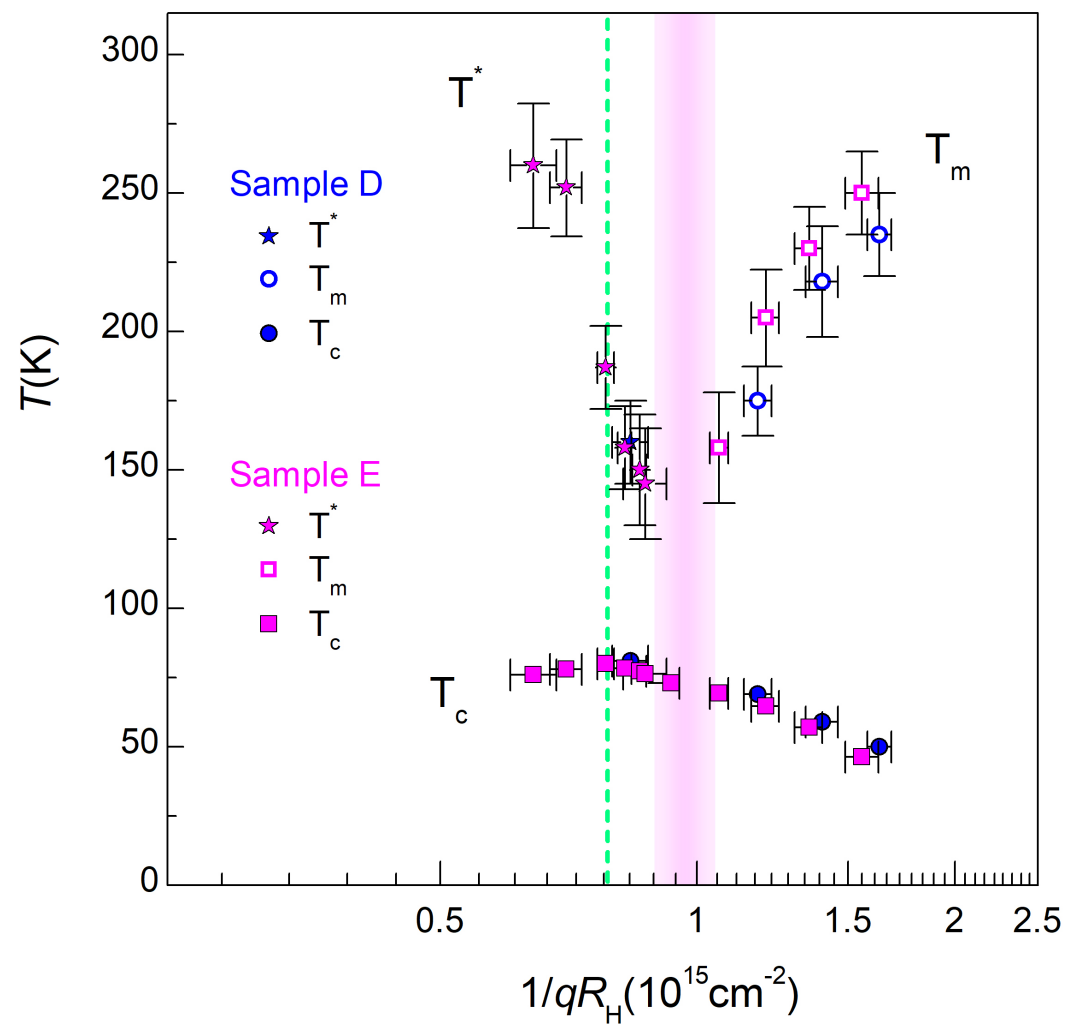

Figure 5: Phase diagram of sample $\mathrm{E}$ and $\mathrm{D}$ with doping inferred from $R_{\mathrm{H}}$. Characteristic temperatures $T^{*}, T_{\mathrm{m}}$ and $T_{\mathrm{c}}$ as a function of $1 / q R_{\mathrm{H}}$. The $1 / q R_{\mathrm{H}}$ scale is logarithmic to account for an exponential relation between the carrier density determined in this manner and that determined by the empirical formula. The dashed vertical line indicates the optimal doping level, namely the $1 / q R_{\mathrm{H}}$ value corresponding to the maximum $T_{\mathrm{c}}$ measured. The violet band indicates the critical region corresponding to the low temperature strange metal phase.

optimal doping ( 0.16 holes $/ \mathrm{Cu}$ ) and of \pm 0.001 holes $/ \mathrm{Cu}$ around 0.18 holes $/ \mathrm{Cu}$. For higher doping the error becomes negligible. Hence, as the critical region is close to the top of the dome, one could expect a larger error in the determination of the critical doping with the empirical formula. It is then reassuring to see that the crossovers and the critical region as deduced from these two estimations of the doping level are similar.

As mentioned earlier, in BSCCO-2212, ground state electronic structure which is effectively masked in part by the superconducting state, could prove to be complex. In some interpretations the quantum critical point is associated with a Lifshitz quantum phase transition, where as a function of increasing doping the hole-like Fermi surface becomes electron-like at 
a critical doping of about 0.19 holes/Cu, as observed in ARPES spectroscopy and theoretical calculations $[15,41,42]$.

In another cuprate superconductor, $\mathrm{YBa}_{2} \mathrm{Cu}_{3} \mathrm{O}_{y}$, the use of very high magnetic fields to suppress superconductivity has allowed to measure a change in carrier density $n_{\mathrm{H}}=1 / q R_{\mathrm{H}}$, from $n_{\mathrm{H}}=1+p$ at high doping to $n_{\mathrm{H}}=p$ at low doping [21], as a further confirmation of change of the Fermi surface topology at quantum criticality in cuprate compounds [43-45].

\section{CONCLUSIONS}

In this work we identify the critical doping regime in 1 unit cell thick 2D BSCCO-2212 by delimiting the strange metal phase to low temperatures just above the superconducting dome using transport measurements and in-situ electrostatic doping in a high quality low disorder device. Searching for this region in the normal state where the pseudogap, strange metal and Fermi liquid phases appear to meet and by fine-tuning the carrier density in the slightly overdoped regime, where experimental results are scarce, we found it to be centered at about $\sim 0.19$ holes $/ \mathrm{Cu}$ and confined in the range $\sim 0.181-0.195$ holes $/ \mathrm{Cu}$. Our transport data allows comparison with spectroscopic measurements and establishes bounds for the range of doping where a quantum critical point could exist though we do not access this region as it is masked by superconductivity. Our results demonstrate the potential of space charge doping to develop fundamental understanding of superconductivity and phase transitions in high-temperature superconductors and open new perspectives in the field of fundamental research.

We thank the Institut des NanoSciences de Paris for access to the electromagnet facility. We acknowledge the Consortium des salles blanches d'Ile de France, M. Rosticher and J. Palomo for access to clean room facilities. This work was supported by French state funds

managed by the ANR within the Investissements d'Avenir programme under reference ANR11-IDEX-0004-02, and more specifically within the framework of the Cluster of Excellence MATISSE of Sorbonne Université .

\footnotetext{
* abhay.shukla@upmc.fr
} 
[1] Damascelli A, Hussain Z and Shen Z 2003 Rev. Mod. Phys. 75, 473

[2] Barišić N, Chan M K, Li Y, Yu G, Zhao X, Dressel M, Smontara A, and Greven M 2013 Proc Natl Acad Sci U S A 110, 12235

[3] Ando Y, Komiya S, Segawa K, Ono S, and Kurita Y 2004 Phys. Rev. Lett. 93, 267001

[4] Varma C M 1999 Phys. Rev. Lett. 83, 3538

[5] Laughlin R B, Lonzarich G G, Monthoux P, and Pines D 2001 Advances in Physics 50, 361

[6] Li L, Wang Y, Komiya S, Ono S, Ando Y, Gu G D, and Ong N P 2010 Phys. Rev. B 81, 054510

[7] Doiron-Leyraud N et al. 2017 Nat. Commun. 8, 2044

[8] Sachdev S 2010 Phys. Status Solidi B 247, 537

[9] Keimer B, Kivelson S, Norman M, Uchida S, and Zaanen J 2015 Nature 518, 179

[10] Cooper et al. 2009 Science 323, 603

[11] Chang J, Månsson M, Pailhès S, Claesson T, Lipscombe O nJ, Hayden S M, Patthey L, Tjernberg O, Mesot J 2013 Nat. Commun. 4, 2559

[12] Daou R, Doiron-Leyraud M, LeBoeuf D, Li S Y, Laliberté O J, Cyr-Chaunière O, Jo Y J, Balicas L, Yan J -Q, Goodenough J B, Taillefer L 2009 Nature. Phys. 5, 31

[13] Matt et al. 2015 Phys. Rev. B 92, 134524

[14] Vishik L M et al. 2012 Proc Natl Acad Sci U S A 109, 18332

[15] Benhabib S et al. 2015 Phys. Rev. Lett. 114, 147001

[16] Dipasupil R M, Oda M, Momono N, and Ido M 2002 J. Phys. Soc. Jpn. 71, 1535

[17] Ozyuzer L, Zasadzinski J F, Gray, K E, Kendziora C, and Miyakawa N 2002 Europhys. Lett. $\mathbf{5 8}, 589$

[18] Miyakawa N, Zasadzinski J F, Ozyuzer L, Guptasarma P, Hinks D G, Kendziora C, and Gray K E 1999 Phys. Rev. Lett. 83, 1018

[19] 2000 Phys. Rev. Lett. 84, 5848

[20] Mangin-Thro L, Sidis Y, Bourges P, De Almeida-Didry S, Giovannelli F, and Laez-Monot I 2014 Phys. Rev. B 89, 094523

[21] Badoux et al. 2016 Nature 531, 210

[22] Grissonnanche et al. 2014 Nat. Commun. 5, 3280

[23] Presland M, Tallon J, Buckley R, Liu R, and Flower N 1991 Physica C: Superconductivity 176, 95 
[24] E. Sterpetti, J. Biscaras, A. Erb, and A. Shukla 2017 Nat. Commun. 8, 2060

[25] Bouvier J and Bok J 2010 Advances in Condensed Matter Physics 2010, 472636

[26] Konstantinovic Z, Li Z, and Raffy H 2000 Phys.l Rev. B 62, R11989

[27] Bok J and Bouvier J. 2004 Physica C: Superconductivity 403, 263

[28] Biscaras J, Chen Z, Paradisi A, and Shukla A 2015 Nat. Commun. 6, 8826

[29] Paradisi A, Biscaras J, and Shukla A 2015 Appl. Phys. Lett. 107, 143103

[30] Hossain M A et al. 2008 Nat. Phys. 4, 527

[31] Bollinger A T, Dubuis G, Yoon J, Pavuna D, Misewich J, Bozovic I 2011 Nature 472, 458

[32] Drozdov I K, Pletikosic I, Kim C -K, Fujita K, Gu G D, Seamus Davis J C, Johnson P D, Bozovic I, Valla T 2018 Nat. Commun. 9, 5210

[33] Shukla A, Kumar R, Mazher J, and Balan A 2009 Solid State Commun. 149, 718

[34] Balan et al. 2010 J. Phys. D. Appl. Phys. 43, 374013

[35] Bruin J A N, Sakai H, Perry R S, and Mackenzie A P 2013 Science 339, 804

[36] 1999 Physica C: Superconductivity 341-348, 859

[37] Kubo Y, Shimakawa Y,Manako T and Igarashi H 1991 Phys. Rev. B 43, 7875

[38] Naqib S, Cooper J, Tallon J and Panagopoulos C 2003 Physica C: Superconductivity 387, 365

[39] Horio et al. 2018 Phys. Rev. Lett. 4, 077004

[40] Licciardello S, Buhot J, Lu J, Ayres J, Kasahara S, Matsuda Y, Shibauchi T, Hussey N E 2019 Nature 567, 213

[41] Kaminski A, Rosenkranz S, Fretwell H M, Norman M R, Randeria M, Campuzano J C, Park J -M, Li Z Z, and Ray H 2006 Phys. Rev. B 73, 174511

[42] Bragança H, Sakai S, M. C. O. Aguiar M C O and Civelli M 2018 Phys. Rev. Lett. 120, 067002

[43] Hwang H Y, Batlogg B, Takagi H, Kao H L, Kwo J, Cava R J, Krajewski J J, and Peck W F 1994 Phys. Rev. Lett. 72, 2636

[44] Mackenzie A P,Julian S R, Sinclair D C, and Lin C T 1996 Phys. Rev. B 53, 5848

[45] Ando Y, Kurita Y, Komiya S, Ono S, and Segawa K 2004 Phys. Rev. Lett. 92, 197001 